\title{
Effect of Node-Degree Correlation on Synchronization of Identical Pulse-Coupled Oscillators
}

\author{
M. Drew LaMar \\ William \& Mary, mdlama@wm.edu \\ Gregory D. Smith \\ William \& Mary, gdsmit@wm.edu
}

Follow this and additional works at: https://scholarworks.wm.edu/aspubs

Part of the Applied Mathematics Commons

\section{Recommended Citation}

LaMar, M. Drew and Smith, Gregory D., Effect of Node-Degree Correlation on Synchronization of Identical Pulse-Coupled Oscillators (2010). Physical Review E, 81(4).

https://doi.org/10.1103/PhysRevE.81.046206

This Article is brought to you for free and open access by the Arts and Sciences at W\&M ScholarWorks. It has been accepted for inclusion in Arts \& Sciences Articles by an authorized administrator of W\&M ScholarWorks. For more information, please contact scholarworks@wm.edu. 


\title{
Effect of node-degree correlation on synchronization of identical pulse-coupled oscillators
}

\author{
M. Drew LaMar* and Gregory D. Smith ${ }^{\dagger}$ \\ Department of Applied Science, The College of William and Mary, McGlothlin-Street Hall, Williamsburg, Virginia 23187, USA
}

(Received 10 December 2008; revised manuscript received 3 March 2010; published 12 April 2010)

\begin{abstract}
We explore the effect of correlations between the in and out degrees of random directed networks on the synchronization of identical pulse-coupled oscillators. Numerical experiments demonstrate that the proportion of initial conditions resulting in a globally synchronous state (prior to a large but finite time) is an increasing function of node-degree correlation. For those networks observed to globally synchronize, both the mean and standard deviation of time to synchronization are decreasing functions of node-degree correlation. Pulsecoupled oscillator networks with negatively correlated node degree often exhibit multiple coherent attracting states, with trajectories performing fast transitions between them. These effects of node-degree correlation on dynamics of pulse-coupled oscillators are consistent with aspects of network topology (e.g., the effect of node-degree correlation on the eigenvalues of the Laplacian matrix) that have been shown to affect synchronization in other contexts.
\end{abstract}

DOI: 10.1103/PhysRevE.81.046206

PACS number(s): 05.45.Xt, 89.75.- $\mathrm{k}$

\section{INTRODUCTION}

Synchronization is ubiquitous in the biological sciences. In phenomena as diverse as the synchronization of cortical neurons leading to gamma oscillations [1] and gene regulatory networks in populations of quorum sensing bacteria [2], both how the oscillators are coupled-i.e., the network structure - and the dynamics of the oscillators themselves, determine whether or not global synchronization occurs. One of the most extensively studied model systems for synchronization is the phase equations originally proposed by Kuramoto and co-workers [3-5]. Interactions between Kuramoto oscillator phases occur continuously and the resulting dynamics correspond to the long-term behavior of weakly coupled identical limit cycle oscillators.

Analysis of Kuramoto oscillators has addressed many questions regarding network structure and synchronization. Results established in the infinite- $N$ case with all-to-all coupling have elucidated the critical coupling value for the onset to partial synchronization and the stability of the partially coherent and fully coherent states [3]. Restrepo and coworkers $[6,7]$ have considered the finite- $N$ case with more complex network topologies, including directed networks, and established an approximate location for the critical coupling strength and local properties of the bifurcating branch. Jadbabaie et al. [8] have shown the existence of another critical coupling strength beyond which a global synchronous state exists, with its stability given by the eigenvalues of the Laplacian matrix.

Another well-studied model system for synchronization is pulse-coupled oscillators with interactions mediated by instantaneous events with finite magnitude [9-11]. Although not as realistic as integrate-and-fire or Hodgkin-Huxley-style network models, pulse-coupled oscillators are regarded as a reasonable starting point for the study of neural synchronization. While phase-response curves of realistic neuronal

\footnotetext{
*mdlama@wm.edu

†'greg@as.wm.edu
}

models have been well-studied, and synchronization of pulse-coupled oscillators have been explored in a neural context $[9,12]$, comparatively few studies have considered how details of network structure may influence the properties of synchronization of pulse-coupled oscillators $[9,11,13]$.

This paper is focused on how a particular aspect of network structure known as "node-degree correlation" (see Sec. II A) affects the dynamics of synchronization of identical pulse-coupled oscillators. This question is timely because dual intracellular recording and other experimental approaches can provide data on the local connectivity of neuronal networks $[1,14-16]$.

\section{MODEL FORMULATION}

We consider a population of $N$ pulse-coupled oscillators [10] with interconnectivity given by the adjacency matrix of a directed graph, i.e., an $N \times N$ matrix $A$ such that

$$
A_{i j}= \begin{cases}1 & \text { if } i \rightarrow j \\ 0 & \text { otherwise, }\end{cases}
$$

where $i \rightarrow j$ indicates that oscillator $i$ can affect the dynamics of oscillator $j$. While synchronization has been most extensively studied in the context of all-to-all coupled networks $\left(A_{i j}=1\right)$ and networks with reciprocal interactions represented by undirected graphs $\left(A_{i j}=A_{j i}\right)[9,17,18]$, simulation and analysis of synchronization on networks represented by directed graphs are not without precedent $[7,8,11,19]$. In this context of pulse-coupled oscillators, it is convenient to assume no self-interactions $\left(A_{i i}=0\right)$.

\section{A. Network topology and node-degree correlation}

Let the ordered pair $d_{i}=\left(d_{i}^{\text {out }}, d_{i}^{\text {in }}\right)$ denote the out and in degree of the $i$-th oscillator, that is, $d_{i}^{\text {out }}=\sum_{j=1}^{N} A_{i j}$ is the number of oscillators $j$ such that $i \rightarrow j$ and, similarly, $d_{i}^{\text {in }}$ $=\sum_{j=1}^{N} A_{j i}$ is the number of oscillators $j$ such that $j \rightarrow i$. The node-degree correlation [20] of a finite network can be quantified in several ways, perhaps most intuitively using the Pearson correlation coefficient 
(a)

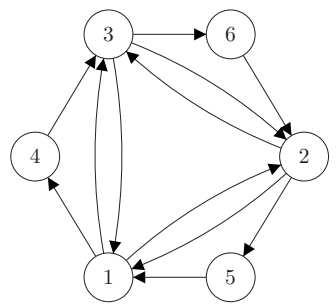

(b)

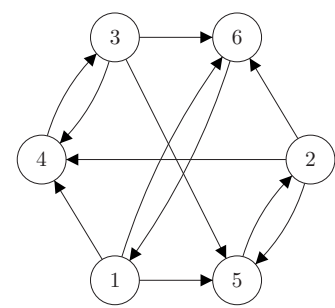

FIG. 1. Example networks with positively (a) and negatively (b) correlated node degree. Degree sequences as in Eq. (2).

$$
\rho=\frac{1}{N} \sum_{i=1}^{N}\left(\frac{d_{i}^{\text {out }}-\mu^{\text {out }}}{\sigma^{\text {out }}}\right)\left(\frac{d_{i}^{\text {in }}-\mu^{\text {in }}}{\sigma^{\text {in }}}\right),
$$

where $\mu^{\text {out }}$ and $\sigma^{\text {out }}$ are the mean and standard deviation of the out-degree over the population of nodes (similarly for $\mu^{\text {in }}$ and $\left.\sigma^{\mathrm{in}}\right)$. For example, Figs. 1(a) and 1(b) show two networks with $N=6$ nodes and degree sequences given by

$$
\begin{aligned}
& \text { (a) }\{(3,3),(3,3),(3,3),(1,1),(1,1),(1,1)\} \\
& \text { (b) }\{(3,1),(3,1),(3,1),(1,3),(1,3),(1,3)\} .
\end{aligned}
$$

While these two networks are similar in many ways (e.g., both have 12 edges), network (a) has a positive node-degree correlation because the Pearson correlation coefficient [Eq. (1)] of it's degree sequence evaluates to $\rho=1$, while network (b) has a negative node-degree correlation $(\rho=-1)$.

Note that the node-degree correlation of a directed network is distinct from the concept of assortativity, which in the context of undirected graphs is essentially the Pearson correlation coefficient of the degrees of pairs of connected nodes (sometimes referred to as edge-degree correlation [20]). Although measures of assortativity can be defined for directed networks using the in and out degree of nodes connected by a directed edge [21], the random networks that are the focus of this study have a wide range of node-degree correlations with negligible edge-degree correlations.

\section{B. Pulse-coupled oscillators}

Following $[10,13]$ and assuming identical intrinsic frequencies, the population of $N$ pulse-coupled oscillators satisfies the phase equations:

$$
\frac{d \phi_{i}}{d t}=1, \quad \text { for } i=1, \ldots, N,
$$

where $\phi_{i} \in[0,1]$. Without loss of generality units of time are chosen so that the natural period of each oscillator is unity. The $i$-th oscillator "fires" when its phase $\phi_{i}$ attains the threshold $\phi_{t h}=1$. When this occurs, the phase of the $i$-th oscillator is reset $\left(\phi_{i} \mapsto 0\right)$ and the phases of oscillators along outgoing connections ( $j$ such that $i \rightarrow j$ ) are advanced according to
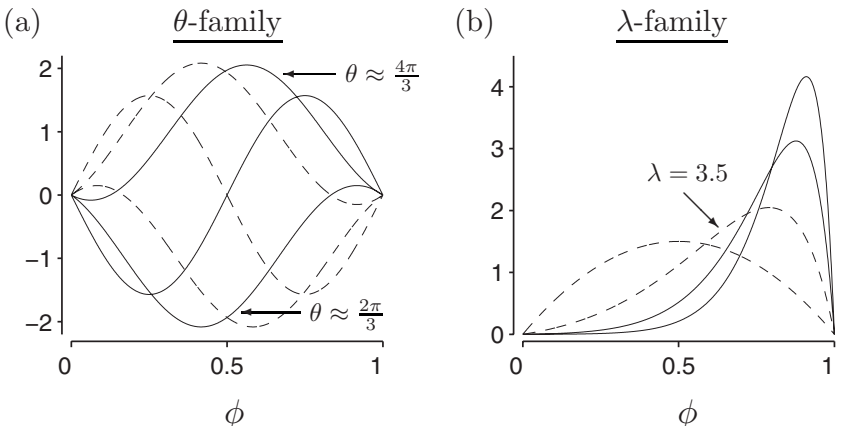

FIG. 2. The $\theta$ and $\lambda$ family of phase-response curves (PRCs) [see Eq. (5)]. Solid and dashed lines denote curves leading to a stable and unstable synchronous state, respectively, in the case of all-to-all coupling. The significance of the labeled curves and arrows is discussed in Sec. III C.

$$
\phi_{j} \mapsto \phi_{j}+\left\{\begin{array}{ll}
\Delta\left(\phi_{j}\right) & \text { for } A_{i j}=1 \\
0 & \text { otherwise }
\end{array},\right.
$$

where $j \neq i$. We will consider three phase-response curves (PRCs) $\Delta(\phi)$ —periodic on the interval $[0,1]$ — given by

$$
\Delta(\phi)=\epsilon \phi,
$$

$$
\begin{gathered}
\Delta(\phi)=\epsilon \alpha_{\theta}[\sin (2 \pi \phi+\theta)-\sin \theta], \\
\Delta(\phi)=\epsilon \alpha_{\lambda} \phi(1-\phi) e^{\lambda \phi},
\end{gathered}
$$

where $0 \leq \theta<2 \pi, \lambda \geq 0$, and $\alpha_{\theta}$ and $\alpha_{\lambda}$ are normalization constants chosen so that $\int_{0}^{1} \Delta(\phi) d \phi=\epsilon \ll 1$. Unless otherwise noted, $\epsilon$ is given by

$$
\epsilon=\frac{k}{\mu^{\text {in }}},
$$

where $k=0.1$ and $\mu^{\text {in }}=\frac{1}{N} \sum_{i=1}^{N} d_{i}^{\text {in }}$ is the mean in degree. This scaling is natural for comparison across networks with different topologies, because the phase advance of the $i$-th oscillator with a characteristic number of incoming neighbors ( $j$ such that $j \rightarrow i$ ) does not depend on the network's degree distribution when these $\mu^{\text {in }}$ neighbors fire simultaneously.

The phase-response curves in Eq. (5) have been chosen for different reasons. PRC 5(a) is the simplest type of excitatory interaction between oscillators that leads to a globally synchronous state for the case of all-to-all connectivity [41]. Numerical experiments with PRC 5(a) show the synchronous state is stable for network topologies that are the focus of this paper (not shown). PRCs 5(b) ( $\theta$ family) and 5(c) ( $\lambda$ family) are more relevant physiologically and satisfy

$$
\Delta(0)=\lim _{\phi \rightarrow 1^{-}} \Delta(\phi)=0
$$

as discussed in [9] (see Fig. 2). The $\theta$ family is similar to the PRCs for the quadratic integrate-and-fire neuron model and radial isochron clock, the latter of which includes regions of phase advance and delay. The $\lambda$ family was used in [9] to fit experimentally observed cortical neuron PRCs that are strictly phase advancing [22]. 
(a)

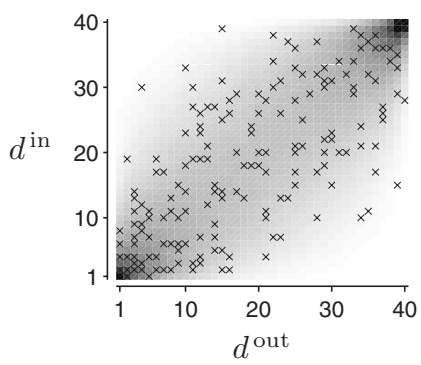

(b)

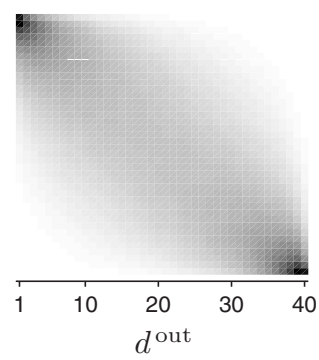

(a)

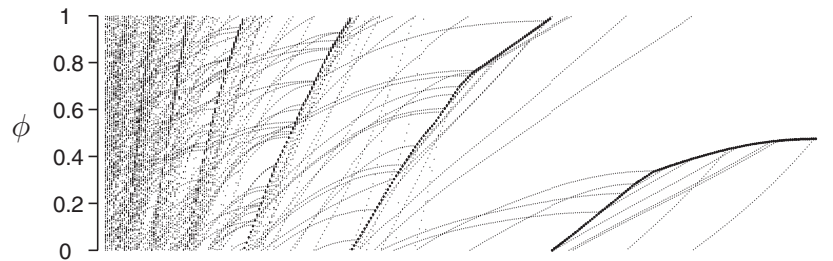

(b)

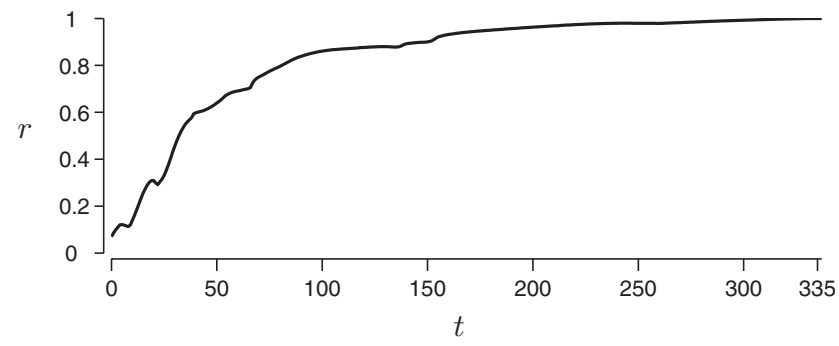

FIG. 4. (a) The stroboscopic phase $\phi$ of 200 pulse-coupled oscillators organized in a network with node-degree correlation of $\rho$ $=0.7$ [see crosses in Fig. 3(a)]. Global synchronization occurs at $t$ $=335$ times the oscillators' natural period. (b) The corresponding coherence measure $r(t)$.

would be appropriate for networks larger than those studied here.

\section{RESULTS}

We will first consider results for the linear PRC in Eq. (5) and verify that similar qualitative results are present over a range of parameter values for the $\theta$ and $\lambda$ family of PRCs in Eqs. (5b) and (5c), respectively. We use the standard global measure of coherence defined as

$$
r(t)=\frac{1}{N}\left|\sum_{j=1}^{N} e^{2 \pi i \phi_{j}(t)}\right|,
$$

nization, we perform numerical experiments on strongly connected networks whose degree sequences- $\left\{d_{i}\right\}_{i=1}^{N}$ where $d_{i}$ $=\left(d_{i}^{\text {out }}, d_{i}^{\text {in }}\right)$ - are drawn from a Gaussian copula [23], that is, a bivariate probability distribution with specified correlation $\rho$ and discrete uniform marginals on the interval $[1,40]$. Panels (a) and (b) of Fig. 3 show the distributions used when the node-degree correlation is $\rho=0.7$ and -0.7 , respectively.

Random networks with specified node-degree correlation were constructed as follows. First, a random degree sequence is drawn from the appropriate Gaussian copula. Next, in- and out-degree pairs of this sequence are replaced at random with new draws from the copula until the sum of the out-degrees equals the sum of the in-degrees. If the resulting degree sequence does not have a network realization [24] a new degree sequence is drawn; otherwise, a network with this degree sequence is constructed using the algorithm of Kleitman and Wang [25]. Finally, to remove any undesired network structure, we perform a Monte Carlo edge-swapping algorithm [26] and check that the resulting network is connected. This procedure well-approximates an independent random draw from the set of networks with the randomly chosen degree sequence. Simpler algorithms for constructing random graphs with specified node-degree correlation [27]

where $r(t) \in[0,1]$. The measure of global synchronization is small $(r \approx 0)$ when the oscillators' phases are broadly distributed, but evaluates to unity $(r=1)$ when the oscillators are globally synchronized. In all simulations that follow, the initial oscillator phases are uniformly distributed on the interval $[0,1]$ and network sizes are fixed at $N=200$ oscillators. Qualitatively similar results are obtained for larger networks (not shown).

Figure 4 shows an example simulation of the dynamics of synchronization in a network of $N=200$ pulse-coupled oscillators in which the correlation between in- and out-degree over the population of nodes is $\rho=0.7$. The degree sequence of the network is illustrated by the crosses superimposed upon the Gaussian copula of Fig. 3(a). Beginning with initial oscillator phases uniformly distributed on the interval $[0,1]$ so that $r(0) \approx 0$ (see above), a globally synchronous state is achieved at $t=335$ (in units of the oscillators' natural period). The gradual agglomeration of clusters of oscillators is revealed by a stroboscopic plot of the evolving oscillator phases [Fig. 4(a)] as well as the standard coherence measure [Fig. 4(b)]. 


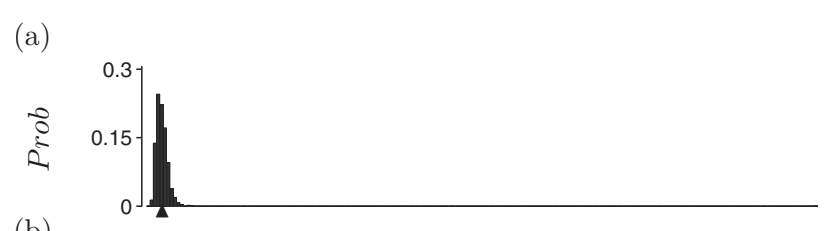

(b)

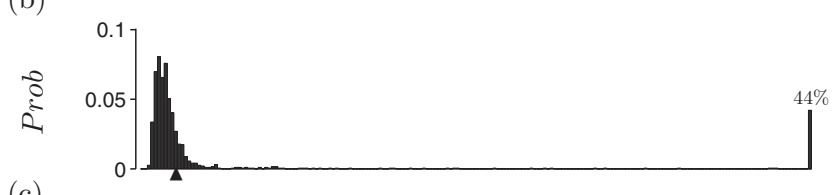

(c)

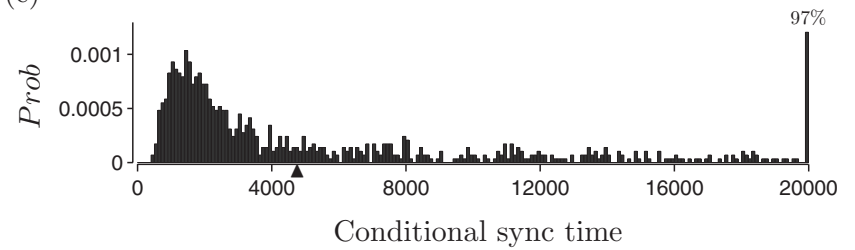

FIG. 5. Histograms of the synchronization time for random networks of pulse-coupled oscillators with specified node-degree correlation (a) $\rho \approx 1$, (b) $\rho=0$, and (c) $\rho \approx-1$. Filled triangles show mean synchronization time of 1000 networks that achieved global synchrony prior to $t=20,000$. Rightmost bar indicates percentage of networks that did not achieve global synchrony.

\section{A. Node-degree correlation and global synchronization}

To explore the effect of node-degree correlation on both the size of the basin of attraction for the globally synchronous state and the time until global synchrony, we repeated simulations similar to Fig. 4 for a large number of random networks with in- and out-degree correlations of $\rho \approx-1, \rho$ $=0$, and $\rho \approx 1$ (constructed as in Sec. II C). While all of the networks with positively correlated node degree $(\rho \approx 1)$ were observed to synchronize, only $56 \%$ of networks with uncorrelated node degree $(\rho=0)$ and $3 \%$ of networks with negatively correlated node degree $(\rho \approx-1)$ synchronized prior to the maximum simulation time of $t=20,000$. Figure 5(a) shows a histogram of synchronization times for the networks with positively correlated node degree $(\rho \approx 1)$ that is focused near the mean value of 460 (filled triangle). Figures 5(b) and 5(c) show similar histograms for the networks with uncorrelated $(\rho=0)$ or negatively correlated $(\rho \approx-1)$ node degree. For those networks observed to synchronize prior to the maximum time of $t=20,000$, the mean synchronization time is larger than that observed for networks with positively correlated node degree [Fig. 5(a)]. While the mode of these distributions does not depend strongly on node-degree correlation, the heavy tails of the corresponding distributions lead to increased mean synchronization times and increased variances.

Figure 6 shows the mean synchronization time with $95 \%$ confidence intervals for simulations as in Fig. 5 with nodedegree correlations in the range $-1<\rho<1$. The solid and dashed lines correspond to networks observed to synchronize prior to maximum simulation times of $t=20,000$ and 40000 , respectively. These calculations demonstrate a general tendency for networks with positively correlated node degree to synchronize faster than networks with negatively correlated node degree. For networks with negative node-degree corre-

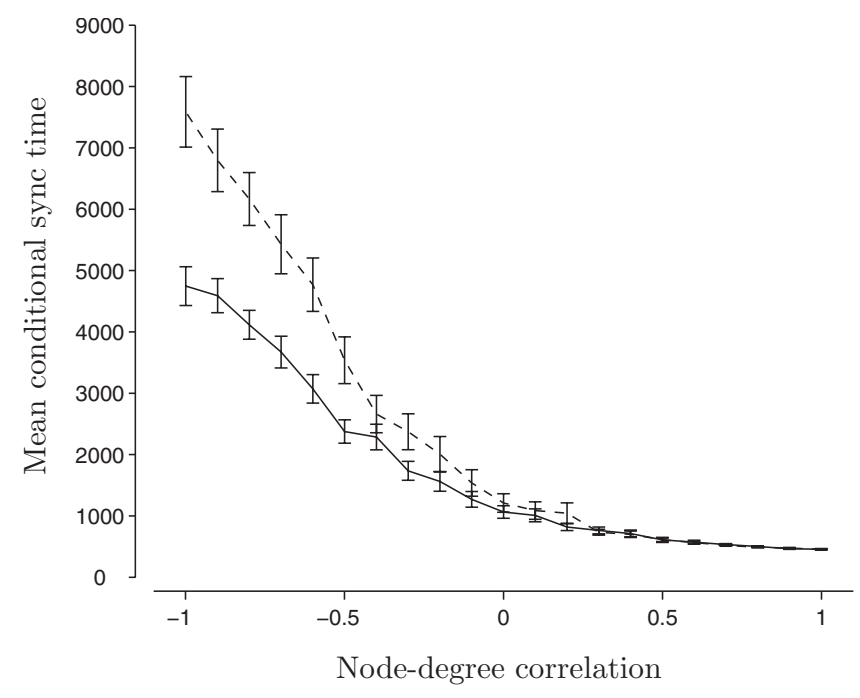

FIG. 6. Mean synchronization time with $95 \%$ confidence intervals for random networks of pulse-coupled oscillators plotted as a function of node-degree correlation. Solid and dashed lines show results for initial conditions that synchronized prior to $t=20,000$ and 40000 , respectively.

lation, increasing the maximum simulation time results in larger mean synchronization times for those networks that synchronize.

Figure 7 shows the proportion of initial conditions that lead to a globally synchronous state as an increasing function of node-degree correlation $(-1<\rho<1)$. Furthermore, for any fixed $\rho$ this proportion does not significantly increase when the maximum simulation time is increased from $t$ $=20,000$ (solid line) to 40000 (dashed line). Given the heavy-tailed distributions for the synchronization time of networks with negatively correlated node degree [Fig. 5(c)], it is possible that the proportion of initial conditions that lead to a globally synchronous state increases sharply for larger

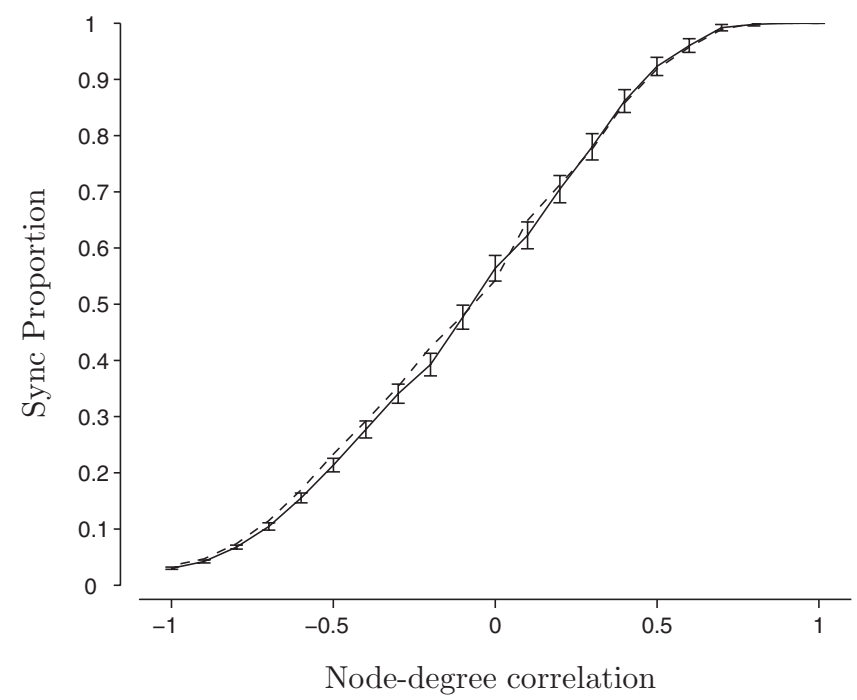

FIG. 7. Proportion of initial conditions leading to global synchrony prior to $t=20,000$ (solid line and 95\% confidence intervals) and 40000 (dashed line) as a function of node-degree correlation of random networks of pulse-coupled oscillators. 


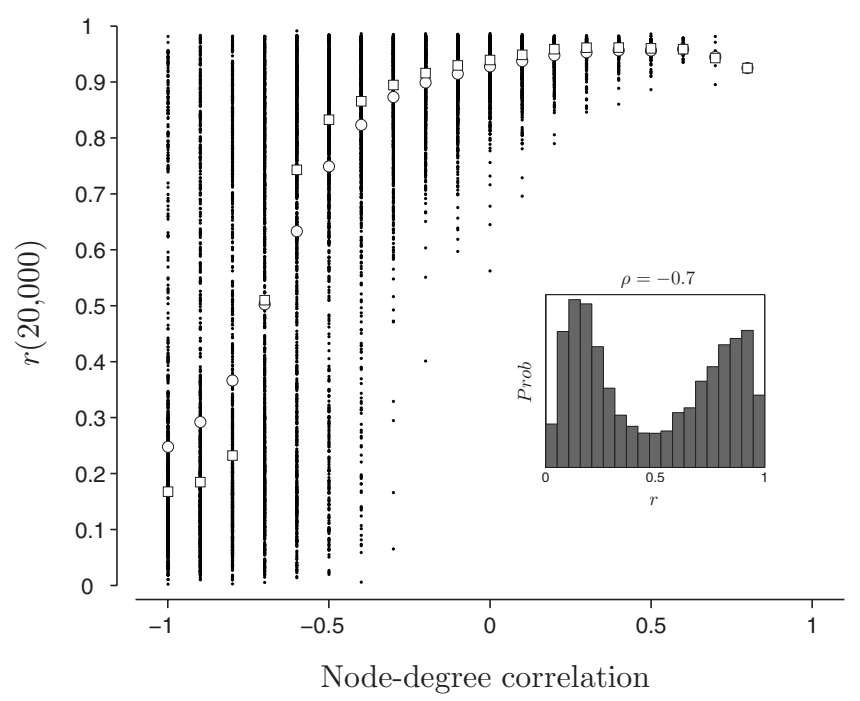

FIG. 8. Scatter plot of synchronization measure $r(20,000)$ as a function of node-degree correlation for networks that did not synchronize prior to $t=20,000$. The open circles and squares denote the mean and median, respectively. Inset shows distribution of synchronization measures among networks with $\rho=-0.7$.

values of $t$ that are not shown here. Nevertheless, the agreement of these two curves suggests that the basin of attraction for the global synchronous state is significantly smaller for networks with negatively correlated node degree.

\section{B. Node-degree correlation and coherence}

Figure 8 shows the synchronization measures $r$ for networks that have not synchronized prior to the maximum simulation time of $t=20,000$ versus node-degree correlation. The mean (open circles) and median (open squares) coherence are shown as a function of $\rho$. As the node-degree correlation decreases, the global synchrony measure is more broadly distributed with mean and median values of $r$ decreasing. The fraction of networks yielding low coherence values increases dramatically as the node-degree correlation is decreased beyond $\rho \approx-0.5$. A bimodal distribution of coherence values is observed for networks with node-degree correlation in the range $-1<\rho<-0.5$. For networks with node-degree correlation of $\rho=-0.7$, for example, the distribution of coherence values is bimodal, nearly symmetric, and has peaks at $r \approx 0.1$ and 0.9 (inset). Figure 9 shows trajectories from three different networks that contributed to the inset histogram in Fig. 8. Panels (a) and (b) show trajectories with a low and high-coherence value, respectively, corresponding to the two modes in the histogram. Panel (c) is included to show that more interesting dynamics may occur, with the existence of two attracting coherent states (plateaus at $r \approx 0.6$ and 0.7 ) with multiple fast transitions between them.

Figure 10 explores the relationship between coherence and coupling strength $[k$ in Eq. (6) $]$ in networks of pulsecoupled oscillators. Using a representative network with node-degree correlation of $\rho \approx 1$, Fig. 10(a) shows synchronization measure $r$ (averaged over 100 trials with different (a)

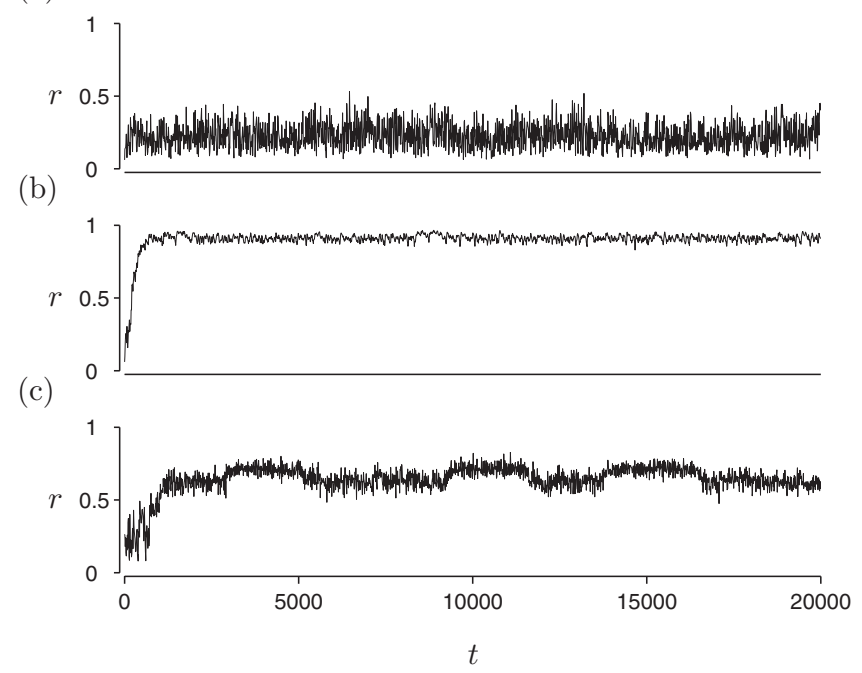

FIG. 9. Synchronization measure $r(t)$ for three $\rho=-0.7$ networks that did not synchronize prior to $t=20,000$ and thus contributed to the histogram in Fig. 8 (inset).

initial conditions) as a function of coupling strength $k$ at $t=10,000$ (circles and solid line), 40000 (squares and dashed line), and 160000 (diamonds and dotted line); filled symbols indicate that all 100 initial conditions achieved global synchrony (i.e., $r=1$ ). In the network with positively correlated node degree, global synchrony is obtained prior to $t=10,000$ regardless of initial conditions so long as the coupling is sufficiently strong $\left(k>3 \times 10^{-3}\right)$.

Figures 10(b) and 10(c) show similar results obtained using representative networks with node-degree correlation

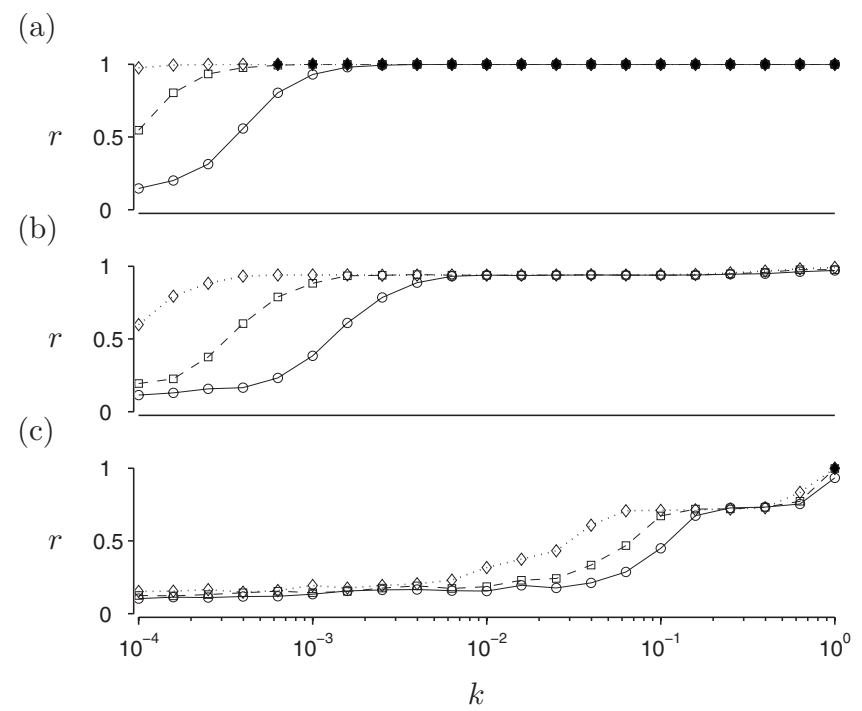

FIG. 10. The synchronization measure $r$, averaged over 100 trials with different initial conditions, as a function of coupling strength $k$ [see Eq. (6)] for representative networks with nodedegree correlation of (a) $\rho \approx 1$, (b) $\rho=0$, and (c) $\rho \approx-1$. Snapshots are shown at three times: $t=10,000$ (circles and solid line), 40000 (squares and dashed line), and 160000 (diamonds and dotted line). Filled symbols indicate global synchrony $(r=1)$ over all initial conditions. 


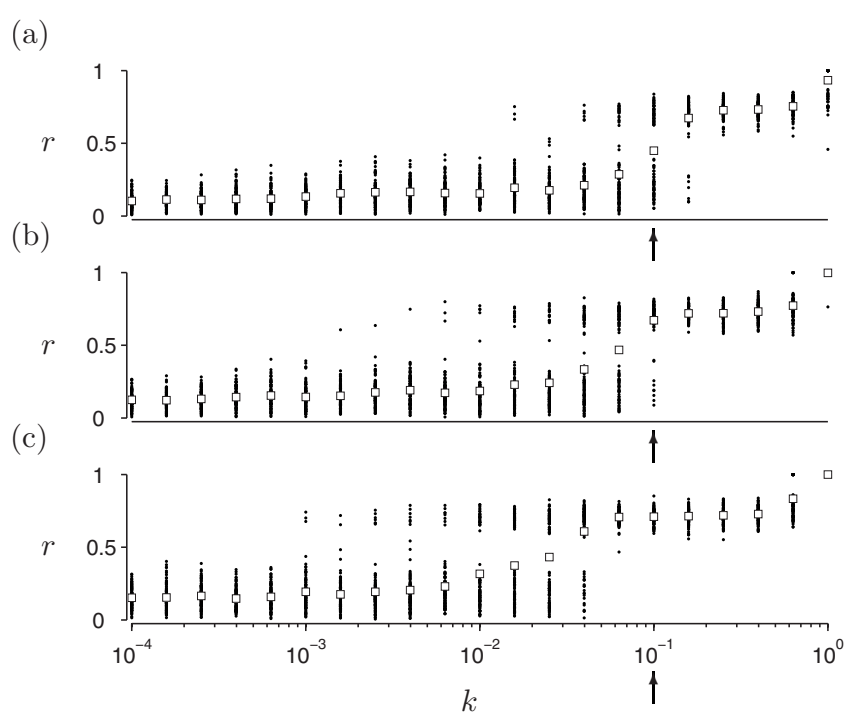

FIG. 11. Scatter plots of the synchronization measure $r$ across trials with different initial conditions for networks with negatively correlated node degree $(\rho \approx-1)$ and a range of coupling strengths $k$. Open squares correspond to average values shown in Fig. 10(c). The arrows at $k=0.1$ correspond to Fig. 12. Snapshots are shown at three times: $t=10,000$ (a), 40000 (b), and 160000 (c).

$\rho=0$ and $\rho \approx-1$, respectively. A comparison of solid, dashed, and dotted lines shows that when oscillators are weakly coupled the networks exhibit long transients with low average coherence, followed by a transition to larger average coherence, the value of which is insensitive to the coupling strength $k$. In particular, the plateaus in Fig. 10(c) suggest the coexistence of high coherence asymptotically stable attracting states and low-coherence states that can trap trajectories for a long period of time before releasing them. Perhaps most importantly, Fig. 10(c) suggests that coupling strengths required for global synchronization are relatively large for random networks with negatively correlated node degree.

Figure 11 shows scatter plots of the synchronization measure $r$ for networks with negatively correlated node degree $(\rho \approx-1)$; the average values (open squares) in Figs. 11(a)-11(c) correspond to the circles, squares, and diamonds, respectively, in Fig. 10(c). As the coupling strength $k$ is varied, plateaus in the average synchronization measure often coincide with clusters of points indicating relatively low variance in the synchronization measure across simulations that are identical save for random initial phases of the oscillators. For values of the coupling strength in transitional regions between plateaus, the synchronization measures observed are more broadly distributed and, for coupling strengths in the approximate range $10^{-2} \leq k \leq 10^{-1}$, two distinct clusters are observed. Comparison of Figs. 11(a)-11(c) (corresponding to $t=10,000,40000$, and 160000 , respectively) shows that these clusters are long-lived.

Figure 12 shows an example time course for a network with negative node-degree correlation $(\rho \approx-1)$ and coupling strength of $k=0.1$ (corresponding to arrows in Fig. 11). The synchronization measure $r$ indicates low coherence until $t$ $\approx 20,000$, at which point the coherence abruptly increases. Qualitatively similar results were obtained for multiple ran-

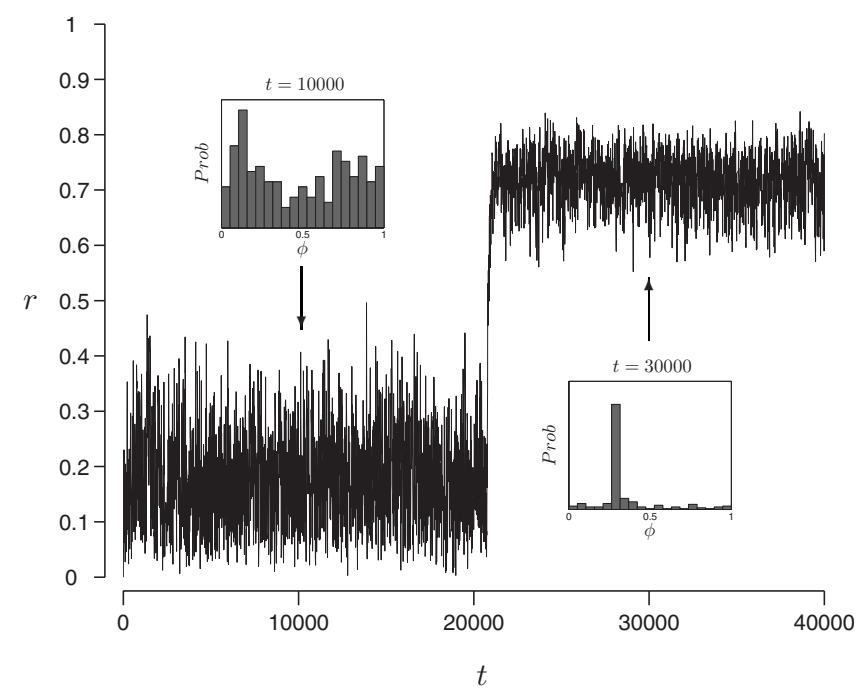

FIG. 12. Time course of synchronization measure $r(t)$ for a network with negatively correlated node degree $(\rho \approx-1)$ and coupling strength $k=0.1$ (cf. arrows in Fig. 11). Insets show distribution of oscillator phases at $t=10,000$ and 30000 .

dom initial conditions. Among these simulations, the time of the abrupt increase in coherence varied widely, while the time-averaged coherence value during the plateaus was nearly constant.

\section{Physiologically realistic PRCs}

We now consider whether node-degree correlation effects the synchronization properties of pulse-coupled networks with physiologically realistic PRCs, specifically, the $\theta$ and $\lambda$ family presented in Fig. 2.

Figures 13(a) and 13(b) show the mean synchronization measure $r(t=20,000)$ for 100 node-degree correlated networks $(\rho \approx 1)$ as a function of the coupling strength $k$ and the PRC shape parameters $\theta$ and $\lambda$, respectively. Using the conditions presented in [9], the dashed curves separate regions of stability and instability of the synchronous state under the assumption of all-to-all connectivity (see legend). For the $\theta$ family of PRCs, the high-coherence region is contained within the stable region $(\pi / 2 \leq \theta \leq 3 \pi / 2)$, in spite of the fact that the networks are not all-to-all coupled. This is not the case for the $\lambda$ family of PRCs, for which the stable region is to the right of the dashed curve.

To illustrate the effect of node-degree correlation on synchronization, Figs. 13(c) and 13(d) give the absolute difference of the mean synchronization measure $r(t=20,000)$ calculated from networks with $\rho \approx 1$ and $\rho \approx-1$. Consistent with results presented above using the linear PRC, there are parameter regions for the $\theta$ and $\lambda$ family of PRCs for which the synchronization properties of networks with anticorrelated node degree are markedly different from networks with correlated node-degree. The PRCs that maximize this difference when the coupling strength is $k=0.1$ are shown in Fig. $2(\theta \approx 2 \pi / 3, \theta \approx 4 \pi / 3, \lambda=3.5)$.

To explore more fully the effect of node-degree correlation on synchronization in the context of these physiologically realistic PRCs, Figs. 14(a) and 14(b) show scatter plots 

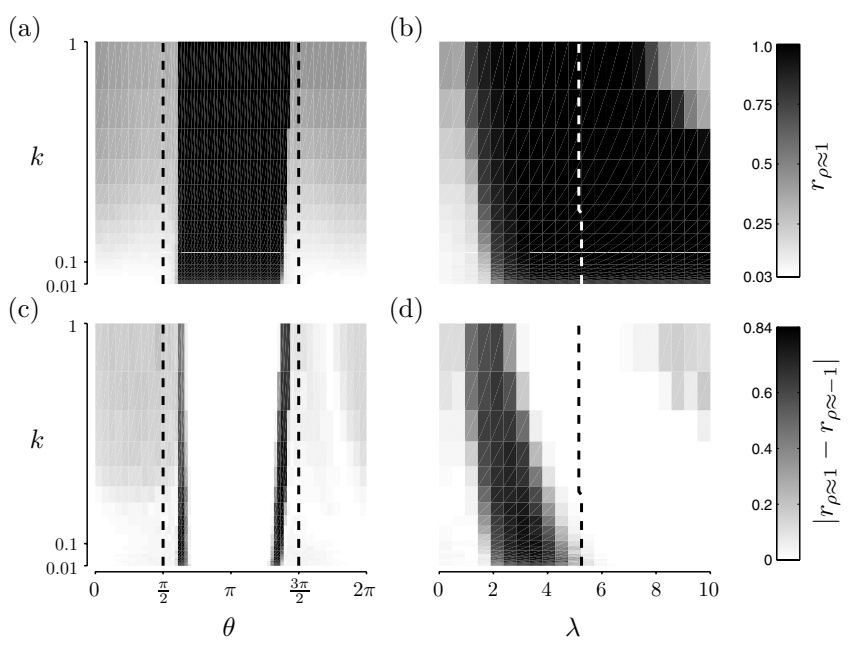

FIG. 13. Top panels show mean synchronization measure $r(t=20,000)$ for 100 node-degree correlated networks $(\rho \approx 1)$ as a function of the coupling strength $k$ and the PRC shape parameters (a) $\theta$ and (b) $\lambda$ (see Fig. 2). Bottom panels show the absolute difference of the mean synchronization measure $r(t=20,000)$ calculated from networks with $\rho \approx 1$ and $\rho \approx-1$. The dashed curves identify regions of stability for the synchronous state under the assumptions of all-to-all connectivity: $\pi / 2 \leq \theta \leq 3 \pi / 2$ (exact), $\lambda>5.25$ (approximate) [9].

of the time to synchronization for networks that synchronized prior to $t=20,000(\theta \approx 4 \pi / 3$ and $\lambda=3.5$, respectively). Mean values are denoted by open circles and are omitted for node-degree correlations that did not result in at least 200 sample points. Consistent with results presented above using the linear PRC, decreasing node-degree correlation is asso-

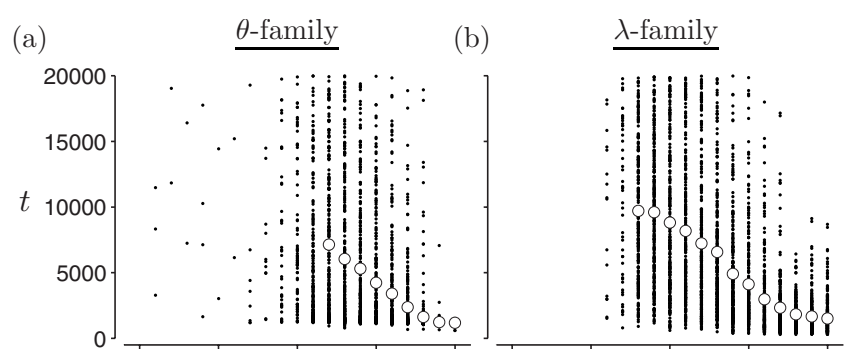

(c)

(d)

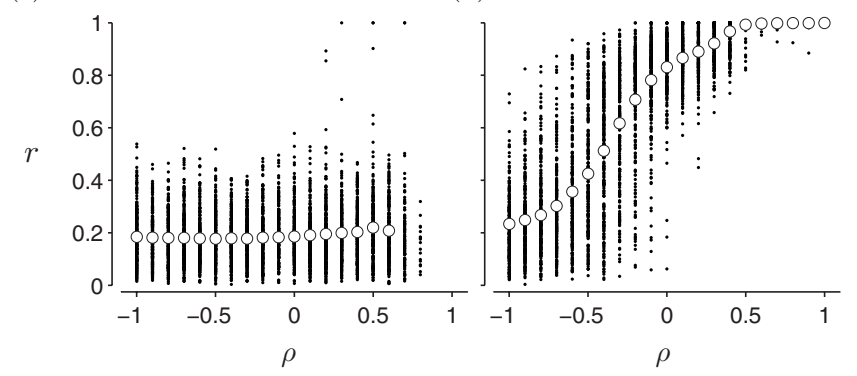

FIG. 14. Top panels show scatter plots of the time to synchronization for networks that synchronized prior to $t=20,000$ using (a) $\theta \approx 4 \pi / 3$ and (b) $\lambda=3.5$ (see Fig. 2). Open circles denote mean values and are omitted for node-degree correlations that did not result in at least 200 sample points. Bottom panels show scatter plots of $r(t=20,000)$ for those networks that did not synchronize prior to $t=20,000$. The coupling strength $k=0.1$.
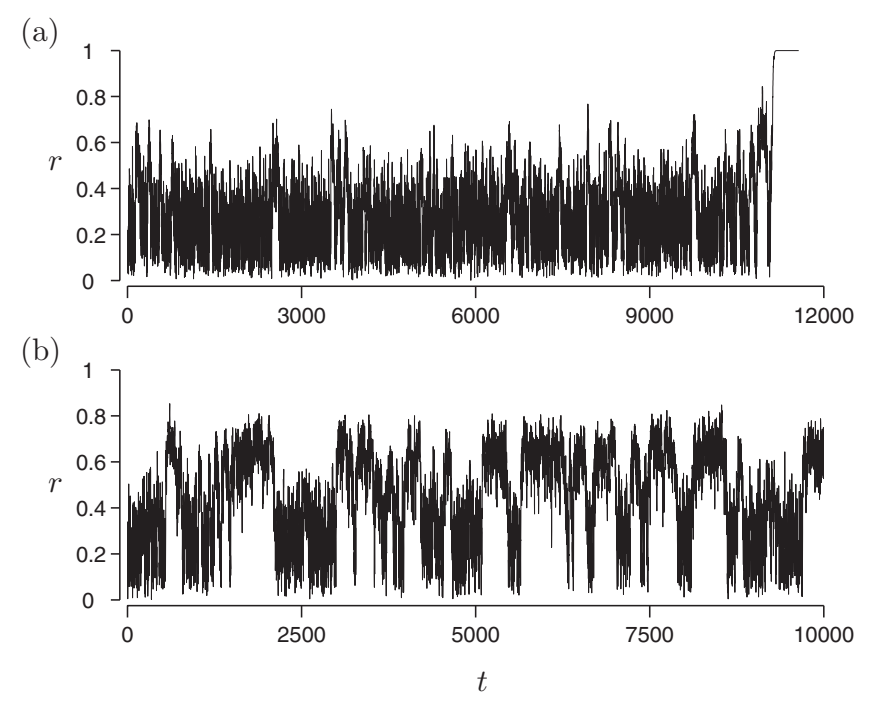

FIG. 15. Representative time courses of the synchronization measure $r(t)$ for networks with negatively correlated node-degree $(\rho \approx-1)$, coupling strength $k=0.275$, and (a) $\theta=4 \pi / 3$ and (b) $\lambda=3.5$ (see Fig. 2).

ciated with longer synchronization times. Numerical evidence suggests the synchronous state is stable for all simulations using the $\theta$-family PRC with $\theta \approx 4 \pi / 3$. However, for the $\lambda$-family PRC with $\lambda=3.5$ there is an increased proportion of simulations resulting in an unstable synchronous state as the node-degree correlation decreases (not shown).

For networks that did not synchronize prior to $t=20,000$, Figs. 14(c) and 14(d) show that decreasing node-degree correlation is associated with smaller coherence for the representative $\theta$ - and $\lambda$-type PRCs. Bimodal distributions of the coherence measure were observed for the $\lambda$-type PRC with node-degree correlation in the range $-0.4 \leq \rho \leq-0.2$ (not shown), similar to the linear PRC (see inset of Fig. 8). While the coherence is a gradually increasing function of nodedegree correlation for the $\lambda$-type PRC (similar to the linear PRC), $r$ is not a strong function of $\rho$ for the $\theta$-type PRC. It is unclear what aspect of these representative PRCs is responsible for this difference.

Figure 15 shows two representative time courses of the synchronization measure $r(t)$ for networks with negatively correlated node-degree $(\rho \approx-1)$ and physiologically realistic PRCs. Figure 15(a) shows a long-lived low coherent state followed by an abrupt transition to global synchrony at $t \approx 11,000(\theta=4 \pi / 3, k=0.275)$. Figure $15(\mathrm{~b})$ shows lowand high-coherence attracting states with many abrupt transitions between them $(\lambda=3.5, k=0.275)$. In both cases qualitatively similar results were obtained using the linear PRC [cf. Figures 9(c) and 12].

\section{DISCUSSION}

We investigated the dynamics of synchronization in random networks of pulse-coupled oscillators with specified node-degree correlation $(-1<\rho<1)$. We found that networks with negatively correlated node degree are less likely to achieve global synchrony (Figs. 5 and 14) and synchro- 
nize more slowly (Fig. 6 and 14) than networks with positively correlated node degree. In networks with negatively correlated node degree, the proportion of initial conditions leading to global synchrony is reduced (Fig. 7 and 14) and the variability of the coherent state is increased (Fig. 8 and 14) in comparison to networks with uncorrelated or positively correlated node degree. Networks with negatively correlated node degree often exhibited interesting dynamics, such as long-lived transients and fast transitions between multiple attracting states, phenomena that were not observed in networks with positively correlated node degree [Figs. 9(c), 11, 12, and 15].

As mentioned in Sec. II A, the node-degree correlation of a directed network is not to be confused with assortativity or edge-degree correlation, concepts most often applied in the context of undirected graphs. While measures of assortativity can be defined for directed networks, the random networks studied here are not significantly edge-degree correlated. While edge-degree correlation of nonlinear oscillators has been studied in the context of Laplacian dynamics [18], to our knowledge this is the first study of the effect of nodedegree correlation in random networks of pulse-coupled oscillators.

\section{A. Initial oscillator phase distribution and synchronization time}

In all simulations presented above, the initial oscillator phases were uniformly distributed on the interval $[0,1]$ leading to small initial coherence $(r(0) \approx 0)$. When networks with negatively correlated node degree are initialized in this fashion, global synchrony is unlikely and synchronization, when it does occur, is slow (Figs. 7 and 14). Nevertheless, it is possible to choose initial conditions with small initial coherence $[r(0) \approx 0]$ that lead to rapid synchronization. For example, if the ordering of initial oscillator phases $\left[\phi_{n}(0)\right.$ $\left.\geq \phi_{n+1}(0)\right]$ coincides with the lexicographical ordering of the node degrees $\left(d_{n}^{\text {out }} \geq d_{n+1}^{\text {out }}\right.$ and $\left.d_{n}^{\text {out }}=d_{n+1}^{\text {out }} \Rightarrow d_{n}^{\text {in }} \geq d_{n+1}^{\text {in }}\right)$, the time to synchronization of networks with $\rho \approx-1$ is shorter than that observed for networks with $\rho \approx 1$ when using the linear and $\theta$-family PRCs $(\theta \approx 4 \pi / 3$, not shown). We found no such effect for the $\lambda$-family of PRCs when $\lambda=3.5$, presumably because the globally synchronous state is unstable (Fig. 13).

\section{B. Node-degree correlation and global network topology}

The Laplacian of an undirected network with adjacency matrix $A$ is defined as $L=D_{A}-A$, where $D_{A}$ is the diagonal matrix of row sums of $A$. The eigenvalues of $L$ give information about the network topology, such as the number of connected components, the community structure, and the rate of mixing of random walkers [28]. In the case of Kuramoto oscillators coupled via an undirected network, the Laplacian provides information about the time to synchronization $[8,29]$. Although such a link has yet to be shown for directed networks of pulse-coupled oscillators, both the undirected and directed Laplacian (defined below) provide information about network topology that may be relevant to synchronization of pulse-coupled systems. (a)

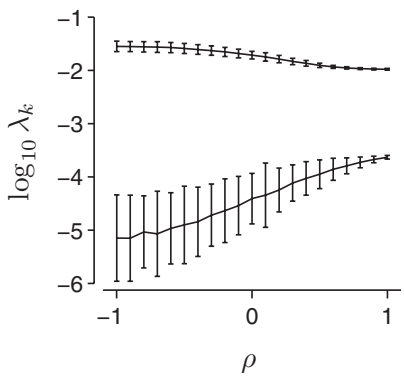

(b)

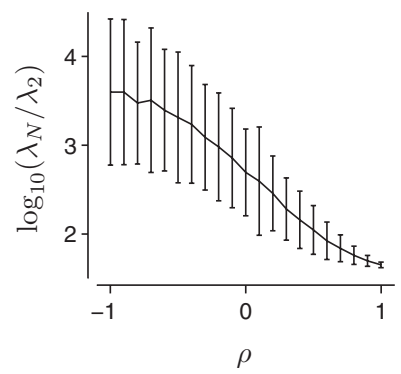

FIG. 16. Laplacian eigenvalues $\lambda_{N}$ (top line) and $\lambda_{2}$ (bottom line) in (a) and ratio $\lambda_{N} / \lambda_{2}$ in (b) as a function of node-degree correlation $(\rho)$. Each point shows mean \pm standard deviation for 1000 random networks.

The Laplacian of a strongly connected directed network can be defined as $[30,31]$

$$
L^{\prime}=D_{\phi}-\frac{D_{\phi} P+P^{T} D_{\phi}}{2},
$$

where $P=D_{A}^{-1} A, A$ is the adjacency matrix for the network, $D_{A}$ is the diagonal matrix of row sums of $A, D_{\phi}$ is a diagonal matrix, and $\phi$ solves $\phi P=\phi$ subject to $\|\phi\|_{1}=1$. Since $L^{\prime}$ is symmetric and has zero row sum, the eigenvalues of $L^{\prime}$ are real, nonnegative, and can be ordered as $0=\lambda_{1}<\lambda_{2} \leq \ldots$. $\leq \lambda_{N}$.

The spectral gap of the Laplacian of a directed network $\left(\lambda_{2}\right)$ has properties similar to the spectral gap of the Laplacian of an undirected network [31]. The spectral gap is known to have an effect on the rate of convergence to the globally synchronous state when identical oscillators are coupled as in the Kuramoto model or via an undirected Laplacian $\left(d \phi_{i} / d t=\omega-\sigma \sum_{j} L_{i j} \phi_{j}\right)[8,29]$. Similarly, the eigenvalue ratio $\lambda_{N} / \lambda_{2}$ is related to the propensity of identical oscillators coupled via an undirected Laplacian to have a stable synchronous state [17]. While it is not known if the directed Laplacian [Eq. (8)] has these properties in the case of pulsecoupled oscillators, it is of interest to explore the effect of node-degree correlation on both $\lambda_{2}$ and the ratio $\lambda_{N} / \lambda_{2}$.

Figure 16 shows the mean Laplacian eigenvalue $\lambda_{2}$ (panel a, bottom line) and the ratio $\lambda_{N} / \lambda_{2}$ (panel b) are monotone increasing and decreasing functions of node-degree correlation, respectively (average over 1000 random networks for each $\rho$ ). Recall that for pulse-coupled networks with negatively correlated node degree (corresponding to small $\lambda_{2}$ and large $\lambda_{N} / \lambda_{2}$ in Fig. 16) we observe slow synchronization (Fig. 6) and reduced percentage of initial conditions that reached global synchronization (Fig. 7). This relationship between Laplacian eigenvalues and synchronization is consistent with prior work focusing on oscillators coupled as in the Kuramoto model or via an undirected Laplacian $[8,17,29]$. Note this correspondence is less clear in the case of the $\lambda$-family PRCs where the synchronous state may be unstable [Fig. 14(b) and 14(d)].

Figure 17 shows the effect of node-degree correlation on three additional measures of network topology: average shortest path length, clustering coefficient, and communica- 
(a)

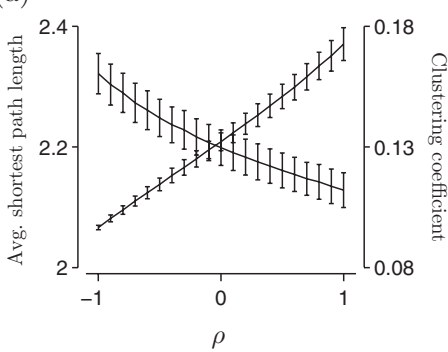

(b)

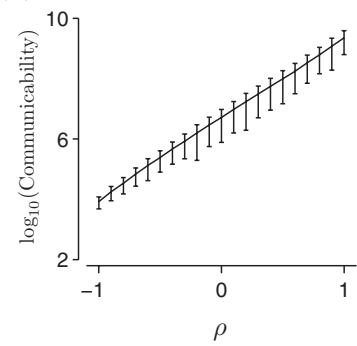

FIG. 17. Three measures of network topology as a function of node-degree correlation: (a) average shortest path length (left axis, decreasing function) and average clustering coefficient (right axis, increasing function); and (b) average communicability. Each point shows mean \pm standard deviation for 1000 random networks.

bility [32]. Figure 17(a) shows that the average shortest path length and clustering coefficient are decreasing and increasing functions of node-degree correlation, respectively, consistent with the known relationship between these measures and synchronization of Kuramoto oscillators on undirected scale-free networks [33]. Figure 18 sheds light on the observed relationship between node-degree correlation and these measures by comparing the adjacency matrices for networks of 200 lexicographically ordered oscillators (Sec. IV A) with $\rho \approx 1$ and $\rho \approx-1$. In particular, the asymmetry present in networks with negatively correlated node degree gives a directional bias to network connectivity that leads to an increased average distance between oscillators and reduced clustering.

\section{Local network topology and the dynamics of synchronization}

To obtain more insight into the dynamics of synchronization in positively and negatively correlated networks, we considered local network properties. For example, Fig. 19 shows how the node-degree correlation of a network influences local clustering of subnetworks composed of nodes with similar in-degree. Local clustering of a subnetwork is defined as the number of arcs divided by the total possible number of arcs. For both positive and zero correlated nodedegree networks, we find the local clustering increases with in-degree. However, for negatively correlated networks, local clustering is a biphasic function of in-degree with maximum

(a)

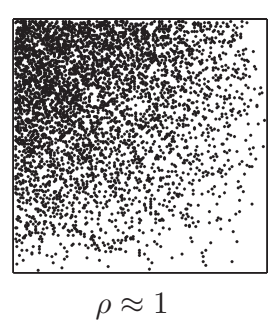

(b)

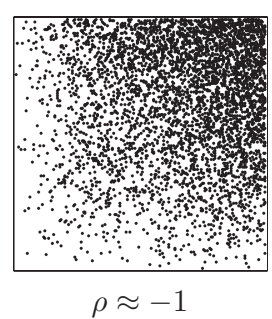

FIG. 18. Adjacency matrices for networks with node-degree correlations (a) $\rho \approx 1$ and (b) $\rho \approx-1$ with lexicographically ordered oscillators (see Sec. IV A).

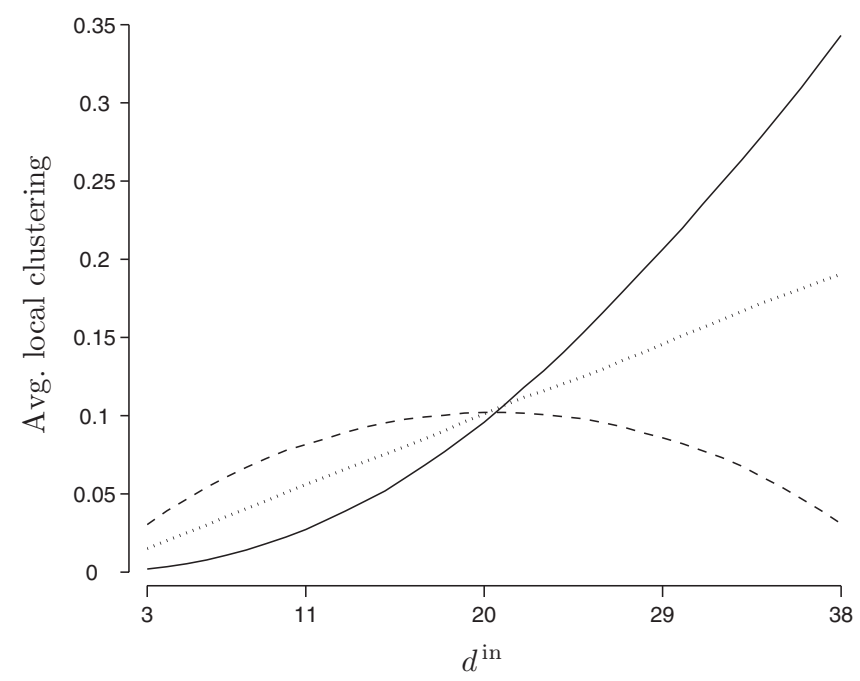

FIG. 19. Average local clustering of subnetworks indexed by in-degree for node-degree correlations of $\rho \approx 1$ (solid), $\rho=0$ (dotted), and $\rho \approx-1$ (dashed). For each $d_{i}^{\text {in }}$ in the range $3 \leq d_{i}^{\text {in }} \leq 38$, a subnetwork was defined by selecting all nodes with in degrees in the window $\left[d_{i}^{\text {in }}-2, d_{i}^{\text {in }}+2\right]$. Error bars suppressed for clarity.

at $d^{\text {in }} \approx 20$. This topological difference between positively and negatively correlated node-degree networks influences the dynamics of synchronization (see below).

Figure 20(a) shows the coherence measure as a function of time calculated on the subnetworks defined above. In this positively correlated network $(\rho \approx 1)$ that achieves synchrony, the larger in-degree nodes synchronize first, consistent with these subnetworks having larger in-degree and larger clustering as in Fig. 19 (solid line). Using the negatively correlated network $(\rho \approx-1)$ that led to the jump seen in Fig. 12, Fig. 20(b) shows higher coherence occurring instead in the subnetworks with $d^{\text {in }} \approx 20$, as well as slightly higher coherence occurring in the subnetworks with higher in-degree (cf. Figure 19). Note that in both cases, the subnetworks with relatively large clustering and large in-degree have higher coherence.

\section{Connections with previous work}

The long-lived transient states observed in networks with negatively correlated node degree (e.g., the $t=10,000$ inset (a)

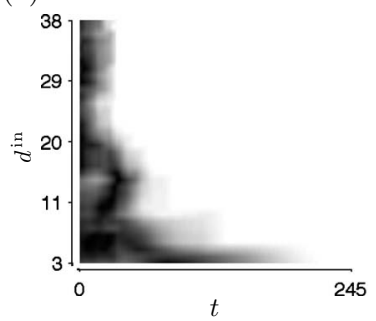

(b)

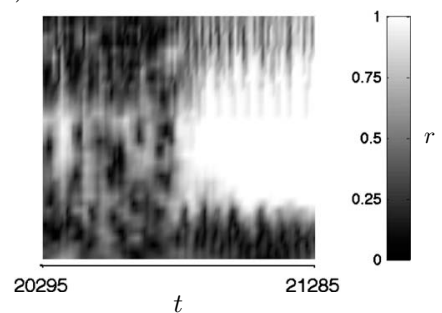

FIG. 20. Synchronization measure $r(t)$ calculated on subnetworks from Fig. 19 illustrating (a) synchronization on a positively correlated network $(\rho \approx 1)$ and $(b)$ the dynamics occurring during the jump illustrated in Fig. 12. 
of Fig. 12) were insensitive to random perturbations of oscillator phases, that is, random perturbations did not immediately lead to a transition from the low-coherence to the high-coherence state, although it does of course change the time at which the jump ultimately occurs (not shown). In prior work that did not consider node-degree correlation, long-lived transient states were observed in networks of identical pulse-coupled oscillators with and without time delay [34-36].

Similar to the results reported here, node-degree correlation has been shown to influence the synchronization of Kuramoto oscillators whose governing equation is given by Eq. (7) with $\Gamma\left(\phi_{i}, \phi_{j}\right)=\epsilon \sin \left(\phi_{j}-\phi_{i}\right)$ and $\epsilon=k / N[6,37,38]$. In particular, the onset of synchronization is shifted to smaller values of the coupling strength in directed networks of Kuramoto oscillators with positively correlated node degree $[7,20]$. Restrepo and co-workers have shown that nodedegree correlation is proportional to the largest eigenvalue of the network adjacency matrix (so long as the largest degree is not too large) and, in the case of Kuramoto oscillators, the critical coupling strength is inversely proportional to the largest eigenvalue. Our observations of the effect of coupling strength and node-degree correlation on synchronization of pulse-coupled oscillators similarly suggest a tendency of the positively correlated networks to synchronize more easily than negatively correlated networks (Fig. 10).

Low-coherence states tend to be associated with heterogeneity in networks of coupled oscillators. For example, synchronization is accelerated in all-to-all coupled networks of Kuramoto oscillators when variability of intrinsic frequency is reduced. Similarly, in random networks of pulsecoupled oscillators, synchronization is promoted by scaling the efficacy of connections so that each oscillator receives the same input when its incoming neighbors fire [34,36]. As expected, when this scaling was applied to our networks of pulse-coupled oscillators, mean synchronization time and percent synchronization are no longer strong functions of node-degree correlation. Nevertheless, our observations of a strong effect of node-degree correlation on the dynamics of synchronization (Figs. 6-8 and 14) is not an obvious consequence of heterogeneous network structure, because in our simulations networks with positively and negatively correlated node degree are equally heterogeneous, e.g., the marginal distribution of in- and out-degree is identical in all simulations performed.

\section{ACKNOWLEDGMENTS}

The authors would like to thank the referees for useful comments and suggestions. This material is based upon work supported by the NSF under Grant No. 0443843 to Smith. LaMar was funded by the Howard Hughes Medical Institute Science Education Grant 52005868 to the College of William and Mary. This work was performed using computational facilities enabled by grants from Sun Microsystems and Virginia's Commonwealth Technology Research Fund.
[1] M. Bazhenov, N. F. Rulkov, and I. Timofeev, J. Neurophysiol. 100, 1562 (2008).

[2] J. Garcia-Ojalvo, M. B. Elowitz, and S. H. Strogatz, Proc. Natl. Acad. Sci. U.S.A. 101, 10955 (2004).

[3] J. A. Acebrón, L. L. Bonilla, C. J. Pérez Vicente, F. Ritort, and R. Spigler, Rev. Mod. Phys. 77, 137 (2005).

[4] Y. Kuramoto, Chemical Oscillations, Waves, and Turbulence (Springer, New York, 1984).

[5] S. H. Strogatz, Physica D 143, 1 (2000).

[6] J. G. Restrepo, E. Ott, and B. R. Hunt, Phys. Rev. E 71, 036151 (2005).

[7] J. G. Restrepo, E. Ott, and B. R. Hunt, Chaos 16, 015107 (2006).

[8] A. Jadbabaie, N. Motee, and M. Barahona, Proceedings of IEEE American Control Conference (2004), Vol. 5, pp. 42964301.

[9] P. Goel and B. Ermentrout, Physica D 163, 191 (2002).

[10] R. Mirollo and S. Strogatz, SIAM J. Appl. Math. 50, 1645 (1990).

[11] M. Timme, Ph.D. Thesis, Max-Planck-Institut für Strömungsforschung, 2002.

[12] T.-W. Ko and B. Ermentrout, Phys. Rev. E 79, 016211 (2009).

[13] X. Guardiola, A. Díaz-Guilera, M. Llas, and C. J. Pérez, Phys. Rev. E 62, 5565 (2000).

[14] D. Eytan and S. Marom, J. Neurosci. 26, 8465 (2006).

[15] G. Molnár, S. Oláh, G. Komlósi, M. Füle, J. Szabadics, C. Varga, P. Barzó, and G. Tamás, PLoS Biol. 6, 1842 (2008).
[16] A.-M. M. Oswald and A. D. Reyes, J. Neurophysiol. 99, 2998 (2008).

[17] M. Barahona and L. M. Pecora, Phys. Rev. Lett. 89, 054101 (2002).

[18] M. di Bernardo, F. Garofalo, and F. Sorrentino, Int. J. Bifurcation Chaos Appl. Sci. Eng. 17, 3499 (2007).

[19] C. Wu, Nonlinearity 18, 1057 (2005).

[20] J. G. Restrepo, E. Ott, and B. R. Hunt, Phys. Rev. E 76, 056119 (2007).

[21] G. Zamora-López, C. Zhou, V. Zlatić, and J. Kurths J. Phys. A: Math. Theor. 41, 224006 (2008).

[22] A. D. Reyes and E. E. Fetz, J. Neurophysiol. 69, 1661 (1993).

[23] R. B. Nelsen, An Introduction to Copulas (Springer, New York, 1998).

[24] D. R. Fulkerson, Pac. J. Math. 10, 831 (1960).

[25] D. Kleitman and D. Wang, Discrete Math. 6, 79 (1973).

[26] G. W. Cobb and Y.-P. Chen, Am. Math. Monthly 110, 265 (2003).

[27] F. Chung, L. Lu, and V. Vu, Proc. Natl. Acad. Sci. U.S.A. 100, 6313 (2003).

[28] F. R. K. Chung, Spectral Graph Theory, CBMS Regional Conference Series in Mathematics (American Mathematical Society, Providence, RI, 1997), Vol. 92.

[29] J. Almendral and A. Díaz-Guilera, New J. Phys. 9, 187 (2007).

[30] F. Chung, Ann. Comb. 9, 1 (2005).

[31] C. Wu, Linear Algebra Appl. 402, 207 (2005).

[32] E. Estrada and D. J. Higham, University of Strathclyde Math- 
ematics Research Report No. 17, 1 (2008).

[33] J. Gómez-Gardeñes and Y. Moreno, Int. J. Bifurcation Chaos Appl. Sci. Eng. 17, 2501 (2007).

[34] M. Timme, F. Wolf, and T. Geisel, Phys. Rev. Lett. 89, 154105 (2002).

[35] R. Zillmer, R. Livi, A. Politi, and A. Torcini, Phys. Rev. E 74, 036203 (2006).

[36] A. Zumdieck, M. Timme, T. Geisel, and F. Wolf, Phys. Rev. Lett. 93, 244103 (2004).

[37] J. G. Restrepo, E. Ott, and B. R. Hunt, Phys. Rev. Lett. 97, 094102 (2006).

[38] J. G. Restrepo, E. Ott, and B. R. Hunt, Phys. Rev. Lett. 96, 254103 (2006).

[39] H. Broer, K. Efstathiou, and E. Subramanian, Nonlinearity 21, 1385 (2008).
[40] A. Díaz-Guilera, C. J. Pérez, and A. Arenas, Phys. Rev. E 57, 3820 (1998).

[41] Though not as physiologically relevant as the $\theta$ and $\lambda$ family of PRCs, the linear PRC is a simple functional form that is parameterized solely by the coupling strength $\epsilon$. A linear PRC can be motivated using the Mirollo-Strogatz formalism with position-phase relation $f(\phi)=\log _{a+1}(a \phi+1)$ and the corresponding PRC given by $\Delta(\phi)=\left[(a+1)^{\hat{\epsilon}}-1\right]\left(\phi+\frac{1}{a}\right)$, where $\hat{\epsilon}$ is a small positional displacement (Ref. [39]). If we choose $\hat{\epsilon}=\log _{a+1}(1+\epsilon)$, then we have the affine linear PRC $\Delta(\phi)=\epsilon\left(\phi+\frac{1}{a}\right)$, which is approximated by Eq. (5) when $a$ is large. In addition, PRCs for the leaky integrate-and-fire neuron model are approximately affine linear when the ratio of the oscillator period and membrane time constant is small (Ref. [40]). 